\title{
The formation of the building blocks of peptides on interstellar dust grains
}

\author{
N. F. W. Ligterink ${ }^{1,2} \mathbb{D}$, J. Terwisscha van Scheltinga ${ }^{2}(1)$, \\ V. Kofman ${ }^{2}$, V. Taquet ${ }^{3}$, S. Cazaux ${ }^{4}$, J. K. Jørgensen ${ }^{5}$ (i), \\ E. F. van Dishoeck ${ }^{6,7}$ (i) H. Linnartz ${ }^{2}$ (i) and The PILS team \\ ${ }^{1}$ Center for Space and Habitability, University of Bern, Sidlerstrasse 5, \\ CH-3012 Bern, Switzerland \\ email: niels.ligterink@csh.unibe.ch \\ ${ }^{2}$ Laboratory for Astrophysics, Leiden Observatory, Leiden \\ University, PO Box 9513, 2300 RA Leiden, the Netherlands \\ ${ }^{3}$ INAF-Osservatorio Astrofisico di Arcetri, Largo E. Fermi 5, I-50125, Florence, Italy \\ ${ }^{4}$ Faculty of Aerospace Engineering, Delft University of Technology, NL-2629 HS Delft, \\ the Netherlands \\ ${ }^{5}$ Centre for Star and Planet Formation, Niels Bohr Institute \& Natural History Museum of \\ Denmark, University of Copenhagen, Øster Voldgade 5-7, 1350 Copenhagen K., Denmark \\ ${ }^{6}$ Leiden Observatory, Leiden University, PO Box 9513, 2300 RA Leiden, the Netherlands \\ ${ }^{7}$ Max-Planck Institut für Extraterrestrische Physik (MPE), Giessenbachstr. 1, \\ 85748 Garching, Germany
}

\begin{abstract}
The emergence of life on Earth may have its origin in organic molecules formed in the interstellar medium. Molecules with amide and isocyanate groups resemble structures found in peptides and nucleobases and are necessary for their formation. Their formation is expected to take place in the solid state, on icy dust grains, and is studied here by far-UV irradiating a $\mathrm{CH}_{4}: \mathrm{HNCO}$ mixture at $20 \mathrm{~K}$ in the laboratory. Reaction products are detected by means of infrared spectroscopy and temperature programmed desorption - mass spectrometry. Various simple amides and isocyanates are formed, showing the importance of ice chemistry for their interstellar formation. Constrained by experimental conditions, a reaction network is derived, showing possible formation pathways of these species under interstellar conditions.
\end{abstract}

Keywords. Astrochemistry, methods: laboratory, Astrobiology

\section{Introduction}

Prebiotic molecules are species that resemble functional groups of biogenic molecules and are thought to be involved in the formation of molecules that are relevant to life, such as amino acids, nucleobases and sugars (Herbst \& van Dishoeck 2009). The interstellar presence of prebiotic molecules supports the idea that the building blocks of life may have an extraterrestrial origin, for example glycolaldehyde, the simplest "sugar" (Hollis et al. 2004; Jørgensen et al. 2016). Among these prebiotics, molecules with an amide $(-\mathrm{NH}-\mathrm{C}(\mathrm{O})-)$ or amide-like structure, such as isocyanic acid (HNCO), hereafter generally called amides, are of particular interest because they resemble structures found in peptide bonds and nucleobases. In terrestrial biochemistry amino acids are connected by peptide bonds resulting in long chains which eventually form proteins, the engines of life. Reactions involving molecules with an amide functional group offer alternative pathways to form peptide chains. 
Amides are widespread throughout the ISM. $\mathrm{HNCO}, \mathrm{NH}_{2} \mathrm{CHO}$ (formamide), $\mathrm{CH}_{3} \mathrm{NCO}$ (methyl isocyanate), $\mathrm{CH}_{3} \mathrm{CONH}_{2}$ (acetamide) and $\mathrm{NH}_{2} \mathrm{CN}$ (cyanamide) have all been detected in various interstellar environments, ranging from star forming regions to comets (Rubin et al. 1971; Hollis et al. 2006; Bisschop et al. 2007; Goesmann et al. 2015; Ligterink et al. 2017; Ligterink et al. 2018; Coutens et al. 2018). Due to the high interstellar abundances of $\mathrm{HNCO}$ and $\mathrm{NH}_{2} \mathrm{CHO}$, many studies have been dedicated to elucidating their formation mechanism, both in the solid-state (e.g. Raunier et al. 2004, Jones et al. (2011), Noble et al. 2015) and in the gas-phase (e.g. Barone et al. 2015). Less extensively studied are the formation of the more complex amides (Agarwal et al. (1985), Henderson \& Gudipati 2015), and in particular how their formation pathways are linked is still an open question.

In this proceeding we summarize results from Ligterink et al. 2017 and Ligterink et al. 2018, showing the formation of several amides in far-UV irradiated interstellar ice analogues. Various interlinked radical recombination reactions are shown to be at the basis of the formation of these species.

\section{Experimental}

The formation of amides is studied using the CryoPAD2 set-up (Ligterink et al. 2017; Ligterink et al. 2018). In short, it consists of a central chamber at ultra-high vacuum conditions $\left(P \leqslant 10^{-10} \mathrm{mbar}\right)$. A gold-coated, reflective surface is positioned at the center of the chamber, which is cryogenically cooled to temperatures as low as $12 \mathrm{~K}$. Gases are directly deposited on this surface, forming an ice layer that simulates the ice mantles on interstellar dust grains. The output of a Microwave Discharge Hydrogen-flow Lamp (MDHL, Ligterink et al. 2015) is directed at the surface and used to energetically process the ice with far-UV radiation $(10.2-7.3 \mathrm{eV})$. Gas mixtures of $\mathrm{CH}_{4}$ (Linde Gas, $99.995 \%$ purity) and $\mathrm{HNCO}$ (Sigma-Aldrich, $98 \%$ purity) are prepared in a gas mixing line and deposited on the substrate at $20 \mathrm{~K}$. The ice layers are UV irradiated for 20 minutes, corresponding to a total fluence of $(1.3 \pm 0.1) \times 10^{17}$ photons $\mathrm{cm}^{-2}$, which in turn equals the far-UV exposure in a dark cloud lifetime of about $3 \times 10^{5}$ years, assuming a dark cloud far-UV flux of $10^{4}$ photons $\mathrm{s}^{-1}$. Chemical changes within the ice are monitored by Reflection Absorption IR Spectroscopy (RAIRS) and mass spectrometry in combination with Temperature Programmed Desorption (TPD).

\section{Results and implications}

The left side of Figure 1 presents the IR spectra recorded during the first $10^{17}$ photons impacting on a $\mathrm{CH}_{4}$ :HNCO ice mixture at $20 \mathrm{~K}$, with a mixing ratio of $1: 1$. All spectra are normalized to the $\mathrm{HNCO}$ peak. Three known spectroscopic features of $\mathrm{CO}_{2}, \mathrm{OCN}^{-}$ and $\mathrm{CO}$ (blue) show up during irradiation. Also, two new features become visible around $2300 \mathrm{~cm}^{-1}$ (red lines), which do not show up while processing samples of pure HNCO or $\mathrm{CH}_{4}$. A red shift of about $10 \mathrm{~cm}^{-1}$ of the two features is seen between samples with ${ }^{12} \mathrm{CH}_{4}$ and ${ }^{13} \mathrm{CH}_{4}$. These spectroscopic features are therefore the result of a product formed in the reaction between $\mathrm{CH}_{4}$ and $\mathrm{HNCO}$. These features are found very close to reported $\mathrm{CH}_{3} \mathrm{NCO}$ values (e.g. Sullivan et al. 1994) and therefore assigned as due to this species.

On the right side of Figure 1 the TPD traces between 150 and $300 \mathrm{~K}$ of the main masses of the simplest, or primary, amides that can be formed from $\mathrm{CH}_{4}: \mathrm{HNCO}$ ice mixtures are shown. These masses are $m / z 45$ for $\mathrm{NH}_{2} \mathrm{CHO}, m / z 59$ for either $\mathrm{CH}_{3} \mathrm{C}(\mathrm{O}) \mathrm{NH}_{2}$ or $\mathrm{CH}_{3} \mathrm{NHCHO}$ and $m / z 60$ for $\mathrm{NH}_{2} \mathrm{CONH}_{2}$. The secondary mass channel of HNCO, $m / z$ 42 , is included as well to trace HNCO. A prominent trailing slope of $m / z 42$ between 150 and $300 \mathrm{~K}$, with a superimposed desorption feature at $\sim 210 \mathrm{~K}$ is seen. The trailing slope is due to residual gas of the main HNCO desorption peak at $130 \mathrm{~K}$, while the desorption 

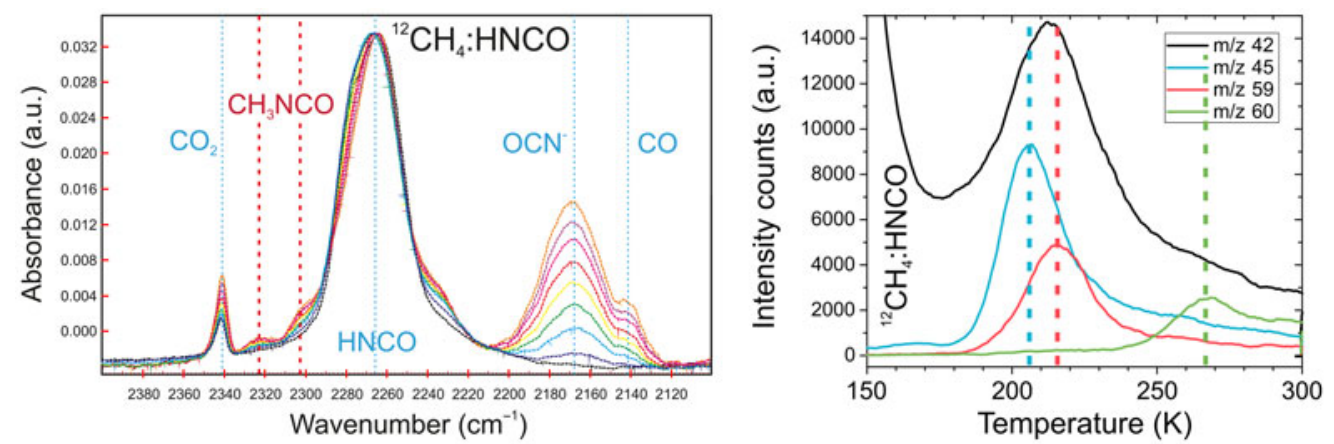

Figure 1. Left: IR spectra of far-UV irradiated $\mathrm{CH}_{4}: \mathrm{HNCO}$ ice mixture (1:1 ratio) at $20 \mathrm{~K}$ over time. Prominent peaks of $\mathrm{OCN}^{-}$and $\mathrm{CO}$ are seen to appear, but also two peaks in the wing of $\mathrm{HNCO}$, which can be assigned to $\mathrm{CH}_{3} \mathrm{NCO}$. Right: TPD-MS trace taken after irradiation of the same $\mathrm{CH}_{4}: \mathrm{HNCO}$ mixture. Desorption of $\mathrm{HNCO}(\mathrm{m} / z$ 42, black $), \mathrm{NH}_{2} \mathrm{CHO}(\mathrm{m} / z 45$, blue), $\mathrm{CH}_{3} \mathrm{CONH}_{2}(m / z 59$, red $)$ and $\mathrm{NH}_{2} \mathrm{CONH}_{2}(m / z 60$, green $)$ is seen.

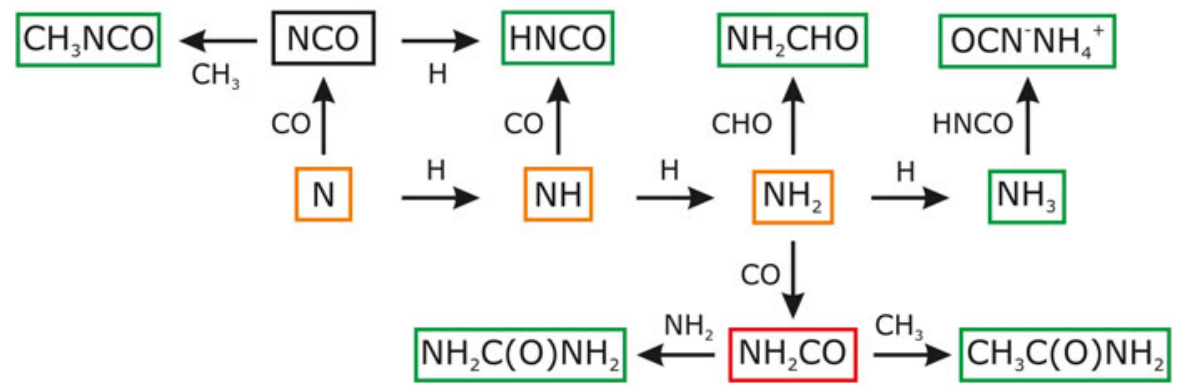

Figure 2. Proposed solid-state formation network of various small amides. The atomic nitrogen hydrogenation chain, eventually leading to $\mathrm{NH}_{3}$ is shown in orange and at the basis of these reactions. Reactions with $\mathrm{CO}$ and $\mathrm{HCO}$ lead to $\mathrm{HNCO}, \mathrm{NH}_{2} \mathrm{CHO}$ and the intermediate radicals $\mathrm{NCO}$ and $\mathrm{NH}_{2} \mathrm{CO}$, while further reactions with $\mathrm{CH}_{3}$ result in $\mathrm{CH}_{3} \mathrm{NCO}, \mathrm{NH}_{2} \mathrm{CONH}_{2}$ and $\mathrm{CH}_{3} \mathrm{CONH}_{2}$.

feature is caused by the thermal decomposition of the $\mathrm{OCN}^{-} \mathrm{NH}_{4}^{+}$salt complex and subsequent desorption of $\mathrm{HNCO}$.

Three desorption peaks of $\mathrm{m} / z 45,59$ and 60 are visible at $\sim 205, \sim 215$ and $\sim 265 \mathrm{~K}$, respectively. $m / z 45$ and 60 do not shift when ${ }^{13} \mathrm{CH}_{4}$ is used and therefore are photoproducts directly resulting from HNCO. They are identified as $\mathrm{NH}_{2} \mathrm{CHO}$ for $\mathrm{m} / z 45$ and $\mathrm{NH}_{2} \mathrm{CONH}_{2}$ for $m / z 60$. When isotopes are used, $m / z 59$ is seen to shift by one mass unit and therefore a product of a reaction between $\mathrm{CH}_{4}$ and $\mathrm{HNCO}$, which realistically can either be $\mathrm{CH}_{3} \mathrm{NHCHO}$ or $\mathrm{CH}_{3} \mathrm{C}(\mathrm{O}) \mathrm{NH}_{2}$. To distinguish between these two products, TPD traces of the pure species are taken and peak desorption temperatures of $184 \mathrm{~K}$ and $219 \mathrm{~K}$ for $\mathrm{CH}_{3} \mathrm{NHCHO}$ and $\mathrm{CH}_{3} \mathrm{C}(\mathrm{O}) \mathrm{NH}_{2}$, respectively, are found. Therefore $\mathrm{m} / z$ is identified as $\mathrm{CH}_{3} \mathrm{C}(\mathrm{O}) \mathrm{NH}_{2}$, aided by fitting its fragmentation pattern with other masses recorded in the TPD (see Ligterink et al. 2018 for details).

In the TPD traces the desorption of higher masses is also seen, which is associated with the formation of more complex species. These masses at $m / z 73,74$ and 88 , are tentatively identified as $\mathrm{CH}_{3} \mathrm{CH}_{2} \mathrm{CONH}_{2}$ (propionamide), $\mathrm{NH}_{2} \mathrm{CH}_{2} \mathrm{CONH}_{2}$ (2-amino acetamide) and $\mathrm{NH}_{2} \mathrm{C}(\mathrm{O}) \mathrm{C}(\mathrm{O}) \mathrm{NH}_{2}$ (oxamide), but secure identification of these species requires more information on their desorption temperatures and fragmentation patterns.

With the data obtained from these experiments and combined with literature data, a solid-state formation network for amides and isocyanates can be derived, see Figure 2 . 
This reaction network starts from the nitrogen $(\mathrm{N})$ hydrogenation chain. $\mathrm{N}, \mathrm{NH}$ and $\mathrm{NH}_{2}$ can react with $\mathrm{CO}$ and $\mathrm{HCO}$ to form $\mathrm{NCO}, \mathrm{HNCO}, \mathrm{NH}_{2} \mathrm{CHO}$ and $\mathrm{NH}_{2} \mathrm{CO}$. Subsequently, the two radicals $\mathrm{NCO}$ and $\mathrm{NH}_{2} \mathrm{CO}$ react with $\mathrm{CH}_{3}$ radicals to form $\mathrm{CH}_{3} \mathrm{NCO}$ and $\mathrm{CH}_{3} \mathrm{CONH}_{2}$. $\mathrm{NH}_{2} \mathrm{CO}$ can also react with $\mathrm{NH}_{2}$ to form $\mathrm{NH}_{2} \mathrm{CONH}_{2}$ and with other radicals to form larger amides.

These experiments clearly show that a large variety of amides can be formed in interstellar ice analogues, starting from simple and abundant species, making it likely that their interstellar formation takes place on icy dust grains. Various amides are intimately linked in their formation, which could explain observational links found between certain molecules, such as $\mathrm{HNCO} / \mathrm{NH}_{2} \mathrm{CHO}$ and $\mathrm{NH}_{2} \mathrm{CHO} / \mathrm{CH}_{3} \mathrm{CONH}_{2}$ (Bisschop et al. 2007, Ligterink et al. 2018, and references therein).

\section{References}

Agarwal, V. K., Schutte, W., Greenberg, J. M., Ferris, J. P., Briggs, R., Connor, S., van de Bult, C. P. E. M., \& Baas, F. 1985, Origins of Life, 16, 1

Barone, V., Latouche, C., Skouteris, D., Vazart, F., Balucani, N., Ceccarelli, C., \& Lefloch, B. 2015, MNRAS, 453, L31

Bisschop, S. E., Jørgensen, J. K., van Dishoeck, E. F., \& de Wachter, E. B. M. 2007, A\&̊A, 465,3

Coutens, A., Willis, E. R., Garrod, R. T., Müller, H. S. P., Bourke, T. L., Calcutt, H., Drozdovskaya, M. N., Jørgensen, J.K., Ligterink, N. F. W., Persson, M. V., Stèphan, G., van der Wiel, M. H. D., van Dishoeck, E.F., \& Wampfler, S. F. 2018, A\&\&A, 612, A107

Goesmann, F., Rosenbauer, H., Bredehöft, J. H., Cabane, M., Ehrenfreund, P., Gautier, T., Giri, C., Krüger, H., Le Roy, L., MacDermott, A. J., McKenna-Lawlor, S., Meierhenrich, U. J., Muñoz-Caro, G. M., Raulin, F., Roll, R., Steele, A., Steininger, H., Sternberg, R., Szopa, C., Thiemann, W., Ulamec, S., et al. 2015, Science, 349, 6247

Henderson, B. L. \& Gudipati, M. S. 2015, ApJ, 800, 1

Herbst, E. \& van Dishoeck, E. F. 2009, ARA $\& A, 47,427$

Hollis, J. M., Jewell, P. R., Lovas, F. J., \& Remijan, A. 2004, ApJ, 613, 1

Hollis, J. M., Lovas, F. J., Remijan, Anthony J., Jewell, P. R., Ilyushin, V. V., \& Kleiner, I. 2006, ApJ, 643, 1

Jones, B. M., Bennett, C. J., \& Kaiser, R. I. 2011, ApJ, 734, 2

Jørgensen, J. K., van der Wiel, M. H. D., Coutens, A., Lykke, J. M., Müller, H. S. P., van Dishoeck, E. F., Calcutt, H., Bjerkeli, P., Bourke, T. L., Drozdovskaya, M. N., Favre, C., Fayolle, E. C., Garrod, R. T., Jacobsen, S. K., Öberg, K. I., Persson, M. V., \& Wampfler, S. F. 2016, $A \& A$, 595, A117

Ligterink, N. F. W., Paardekooper, D. M., Chuang, K.-J., Both, M. L., Cruz-Diaz, G. A., van Helden, J. H., \& Linnartz, H. 2015, A\& A, 584, A56

Ligterink, N. F. W., Coutens, A., Kofman, V., Müller, H. S. P., Garrod, R. T., Calcutt, H., Wampfler, S. F., Jørgensen, J .K., Linnartz, H., \& van Dishoeck, E. F. 2017, MNRAS, 469, 2

Ligterink, N. F. W., Terwisscha van Scheltinga, J., Taquet, V., Jørgensen, J. K., Cazaux, S., van Dishoeck, E. F., \& Linnartz, H. 2018, MNRAS, 480, 3

Noble, J. A., Theule, P., Congiu, E., Dulieu, F., Bonnin, M., Bassas, A., Duvernay, F., Danger, G., \& Chiavassa, T. 2015, A\&A, 576, A91

Raunier, S., Chiavassa, T., Duvernay, F., Borget, F., Aycard, J. P., Dartois, E., \& d'Hendecourt, L. 2004, $A \& A, 416,165$

Rubin, R. H., Swenson, G. W., Jr., Benson, R. C., Tigelaar, H. L., \& Flygare, W. H. 1971, ApJ, 169, L39

Sullivan, J. F., Heusel, H. L., Zunic, W. M., Durig, J. R., Cradock, S., et al. 1994, Spectrochimica Acta Part A: Molecular Spectroscopy, 50, 3 